\title{
Decision Making for Collision Avoidance Systems
}

\author{
Jonas Jansson, Jonas Johansson \\ Volvo Car Corporation \\ Prof. Fredrik Gustafsson \\ Linköping University
}

Copyright (@) 2002 Society of Automotive Engineers, Inc.

\begin{abstract}
Driver errors cause a majority of all car accidents. Forward collision avoidance systems aim at avoiding, or at least mitigating, host vehicle frontal collisions, of which rear-end collisions are one of the most common. This is done by either warning the driver or braking or steering away, respectively, where each action requires its own considerations and design. We here focus on forward collision by braking, and present a general method for calculating the risk for collision. A brake maneuver is activated to mitigate the accident when the probability of collision is one, taking all driver actions into considerations. We describe results from a simulation study using a large number of scenarios, created from extensive accident statistics. We also show some results from an implementation of a forward collision avoidance system in a Volvo V70. The system has been tested in real traffic, and in collision scenarios (with an inflatable car) showing promising results.
\end{abstract}

\section{INTRODUCTION}

It is well known that driver errors are the main cause, or contribute to increased severity, of most accidents. For instance, the Indiana Tri-level (Treat et al. 1979) found driver errors to be a cause or severity-increasing factor in $93 \%$ of the accidents. Furthermore, $27 \%$ of all accidents (USA 1997) were rear-end collisions. This shows the potential of forward collision avoidance (FCA) systems. The crucial part of the algorithm is the decision making, and the conflicting considerations are:

\section{- Avoid all collisions \\ - Never do a faulty intervention}

The design is a compromise between these mutually exclusive conditions. For many reasons, such as driver acceptance of the system and legal requirement that the system itself must not cause hazards, the second condition is the most important one when designing a FCA system.

The final responsibility must always be with the driver, and we stress that the system presented in this paper is driver assistance help.

A further consideration is that such an active system must not brake when the driver can still brake or steer to avoid an accident. This leads to mitigation rather than avoidance system, and in the sequel we refer to the system as Collision Mitigation by Braking (CMBB).

In a combined CMBB and collision mitigation by steering system, CMBB will mainly be activated at low speeds when braking is more efficient.

Algorithms previously proposed in literature for FCA by warning [7][17] and braking [2] are almost exclusively based on relative velocity and relative distance for decision making.

These metrics are easy to understand, but may not be sufficient information in more complex situations such as dense city traffic, situations where the driver switches lane or does hard handling maneuvers.

We here present a method to compute the risk for collision, taking into account measurement uncertainty and driver maneuvers. Decision making is then based on the probability density function for the relative position from the own vehicle to the most dangerous other object for the moment. Similar algorithms have been proposed and exist today for military aircraft, but have, as far as we know, not been studied for road based vehicles.

\section{TARGET TRACKING}

The information sources that are used for FCA systems come from one or more of the following sensors:

-Millimeter radar measuring bearing, range and range rate. 
-IR Radar measuring bearing, elevation, range and range rate.

-Camera with image processing algorithms computing bearing and elevation.

If more than one sensor is used we have a sensor fusion problem, which also includes synchronization in time and space, which is elegantly handled in the Kalman filtering framework described below. The approach is model based using a state space model of the form:

$$
\begin{aligned}
& X_{t+1}=A_{t} X_{t}+B_{t} v_{t}, \operatorname{Cov}\left(v_{t}\right)=Q_{t} \\
& Y_{t}=C_{t} X_{t}+e_{t}, \operatorname{Cov}\left(e_{t}\right)=R_{t}
\end{aligned}
$$

The model should be able to predict how the position of the tracked object evolves in time, and there are a variety of possible models available in the target tracking literature. The one we have chosen is based on the coordinated turn model, where the object is supposed to follow straight line segments and circle segments. This is a fairly good model of roads and typical driver maneuvers, and transitions between different segments are modeled as state noise $v_{t}$.

The state vector is:

$$
X_{t}=\left(\begin{array}{lllll}
x_{t} & y_{t} & v_{x, t} & v_{y, t} & \omega_{t}
\end{array}\right)^{T}
$$

$x_{t}, y_{t}=$ position coordinates in a ground fixed coordinate system at time $t$

$v_{x, t}, v_{y, t}=$ velocity at time $t$

$\omega_{\mathrm{t}}=$ turn rate (yaw rate)

$\theta_{\mathrm{t}}=$ heading angle

The state space matrices are given by

$$
A_{t}(\omega)=\left(\begin{array}{ccccc}
1 & 0 & \frac{a}{\omega t} & \frac{b-1}{\omega_{t}} & 0 \\
0 & 1 & \frac{1-b}{\omega_{t}} & \frac{a}{\omega_{t}} & 0 \\
0 & 0 & b & -a & 0 \\
0 & 0 & a & b & 0 \\
0 & 0 & 0 & 0 & 1
\end{array}\right)
$$

Where:

$$
\begin{aligned}
& a=\sin \left(\omega_{t} T\right) \\
& b=\cos \left(\omega_{t} T\right)
\end{aligned}
$$

$$
B_{t}\left(v_{x}, v_{y}\right)=\left(\begin{array}{ccccc}
0 & 0 & 0 & 0 & 0 \\
0 & 0 & 0 & 0 & 0 \\
0 & 0 & q_{v} \cos (\theta) & 0 & 0 \\
0 & 0 & 0 & q_{v} \sin (\theta) & 0 \\
0 & 0 & 0 & 0 & q_{\omega}
\end{array}\right)
$$

Finally, the measurement equation incorporates the sensor information.

Sensor $i$ gives

$$
\begin{aligned}
& \mathrm{Y}_{\mathrm{i}}(t)=\left(\begin{array}{lll}
R & \dot{R} & \alpha
\end{array}\right) \\
& R=\text { Range } \\
& \dot{R}=\text { Range Rate } \\
& \alpha=\text { Bearing angle }
\end{aligned}
$$

There can be any number of sensors, and they need not be synchronized in time. The idea is to compute the a posteriori probability density function (PDF) of the state vector, given all sensor information up to time t. The model is then used to predict future positions and simulate how the PDF evolves in the near future. From this, we can compute the probability that the relative position belongs to a rectangle $\mathrm{D}$, which is the size of the own vehicle. The posteriori probability density function (PDF) is provided by a model based filter, using the state space model. For linear models, the Kalman filter provides a finite dimensional algorithm to compute this. For non-linear models, such as the one we are using, the so called extended Kalman filter can be used to approximate the PDF, and this is what we have chosen.

Other alternatives include point mass filtering (deterministic numerical integration) and particle filtering (stochastic numerical integration). These may both be too complex for the computational platform we are using, though we have found them to be an interesting alternatives.

The Kalman filtering equations are summarized below:

$$
\begin{aligned}
& \hat{X}_{t+1 \mid t}=A_{t} \hat{X}_{t} \\
& P_{t+1 \mid t}=A_{t} P_{t \mid t} A_{t}^{T}+B_{v, t} Q_{t} B_{v, t}^{T} \\
& \hat{X}_{t \mid t}=\hat{X}_{t \mid t-1}+P_{t \mid t-1} C_{t}^{T}\left(C_{t} P_{t \mid t-1}+R_{t}\right)^{-1}\left(Y_{t}-C_{t} \hat{X}_{t \mid t-1}\right) \\
& P_{t \mid t}=P_{t \mid t-1}-P_{t \mid t-1} C_{t}^{T}\left(C_{t} P_{t \mid t-1} C_{t}^{T}+R_{t}\right)^{-1} C_{t} P_{t \mid t-1}
\end{aligned}
$$

Where: 
$\mathrm{C}_{\mathrm{t}}=\frac{\mathrm{Y}_{\mathrm{i}}(t)}{d X}=\frac{\left(\begin{array}{lll}R & \dot{R} & \alpha\end{array}\right)}{d X}$

$=\frac{\left(R \frac{\Delta X \cdot \Delta v_{x}+\Delta y \cdot \Delta v_{y}}{R} a \tan \left(\frac{\Delta y}{\Delta x}\right)\right.}{d X}=$

$=\left(\begin{array}{ccccc}\frac{\Delta v_{x}}{R}-\frac{\left(\Delta y \cdot \Delta v_{y}+\Delta x \cdot \Delta v_{x}\right) \Delta x}{R^{\frac{3}{2}}} & \frac{\Delta v_{y}}{R}-\frac{\frac{\Delta y}{R}}{-\Delta y} \\ \frac{\left.-\Delta X \cdot \Delta v_{y}+\Delta x \cdot \Delta v_{x}\right) \Delta y}{R^{\frac{3}{2}}} & \frac{\Delta X}{R} & \frac{\Delta y}{R} & 0 \\ \frac{1}{\Delta y^{2}} & \left.\frac{1}{\Delta x^{2}}\right) & 0 & 0 & 0 \\ \Delta X \cdot\left(1+\frac{\Delta y^{2}}{\Delta x^{2}}\right) & & & 0\end{array}\right)$

Here:

$\Delta x=x_{\text {obstacle }}-x_{\text {host }}$

$\Delta y=y_{\text {obstacle }}-y_{\text {host }}$

$\Delta v_{x}=v_{x, \text { obstacle }}-v_{x, \text { host }}$

$\Delta v_{y}=v_{y, \text { obstacle }}-v_{y, \text { host }}$

A thorough treatment of Kalman filtering and model choices is given in [16].

In a collision avoidance application you normally want to evaluate the probability of collision for several future positions. To do this one simply iterates the time update as many times as desired. For autonomous braking actuation we do not need to look further ahead in time than 1.5 seconds (because collisions become unavoidable when they are closer in time). This fact can be realized from calculating the minimum avoidance time from figure 5 .

Other important issues for over all tracking performance such as how to handle multiple tracks (many potentially dangerous objects) and data association will not be discussed in this paper.

\section{RISK ESTIMATION}

The metric we propose for risk estimation is to calculate the probability of collision. This is done by forming the joint PDF of the host's and the other objects' position relative to each other. The probability is calculated by integrating the joint PDF over the area which corresponds to a collision (the area where the two objects physically overlap):

$$
\begin{aligned}
& P(\text { collision })=P\left(\text { Pos }_{\text {Host }}-\text { Pos }_{\text {Object }} \in D\right)= \\
& =\iint_{\text {Pos }_{\text {host }}-P_{o s_{o b j} \in D}} f_{\text {rel }_{\text {p }} \text { pos }}(x, y) d x d y
\end{aligned}
$$

Pos $_{\text {Host }}=$ position of tracked object

Pos $_{\text {Object }}=$ position of tracked object
$D=$ the area which correspond to (geometrical) overlap between the host vehicle and the other object.

$f_{\text {Rel_pos }}(x, y)=$ the PDF of the position of host and obstacle relative to each other

The joint PDF is formed from the PDF of the host's future position and the other obstacles future position. The PDF of the host's and other vehicles' future position can be obtained with one of the filtering methods mentioned above. An example of how the PDFs and the joint PDF look like for one situation is shown in Figure 13. Figure 1 below shows two vehicles meeting. In Figure 2 the PDF for the two vehicles is plotted at 4 different future time instances.

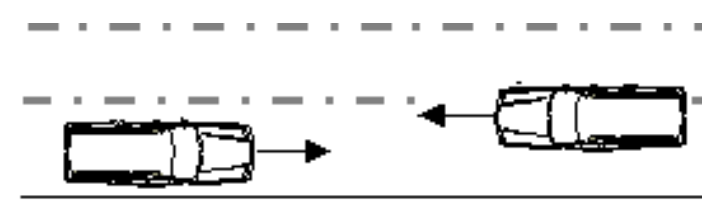

Figure 1. Two cars meeting.

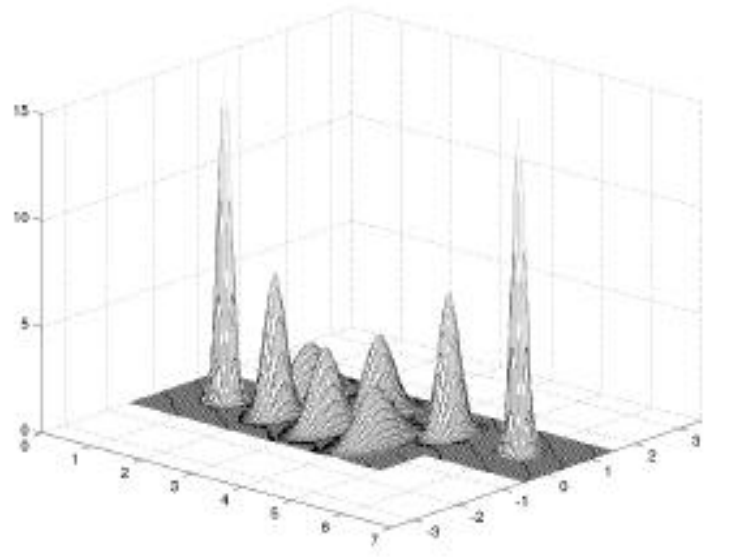

Figure 2. PDF's for the two vehicles in figure 1 at 4 time instances. The narrow peaks correspond to time 1 and the widest peaks correspond to time 4.

In figure 3 the joint probability of the vehicles' position relative to each other is shown. 


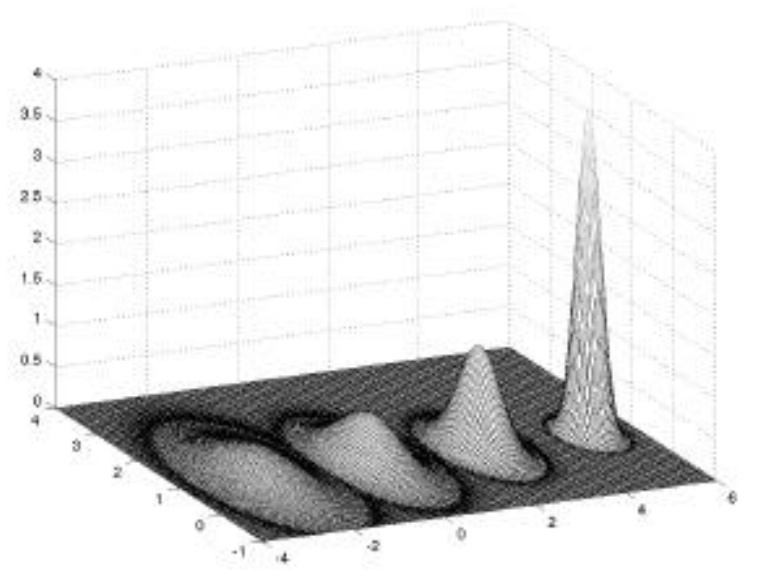

Figure 3. Joint PDF for vehicle's relative position. Again the narrow peak corresponds to time 1 and the widest peak corresponds to time 4 .

Notice that in figure 3 we have switched the coordinate system to one that is fixed to the front of the car coming from the left. At time instance 3 there is a lot of density close to the origin. At this time instance the probability for collision is high.

In our case we get the Kalman filter estimate of $X$ and covariance matrix $\mathrm{P}$ which can be used to compute a confidence region (ellipse) where the relative position will be with a certain probability. Furthermore, with a Gaussian assumption on the PDF, we can compute the risk for collision as described above.

\section{COMPUTATIONAL COMPLEXITY}

Apart from tracking accuracy, computational complexity of the algorithm is also an important issue. There are several considerations concerning the computational complexity of the algorithm. The algorithm has computational complexity of $O(n)$ where $n$ is the number of future time points evaluated. $n$ is a function of how far ahead in time one wants to look and also how close you want the future positions to be in time (= how many future time instances that are calculated). As mentioned above for the CMBB application it is enough to look 1.5 seconds ahead in time. It remains to decide how many points one should calculate for these 1.5 seconds. This is a matter of computational power available, and for the best result one should space the points as close as possible in time. But there is of course a trade-off on how many objects you want to be able to track. The algorithm is $\mathrm{O}(\mathrm{m})$ where we let $\mathrm{m}$ be the number of tracks to be handled. Looking both at future time points and number of tracks the algorithm is $\mathrm{O}\left(\mathrm{n}^{*} \mathrm{~m}\right)$ in computational complexity.

\section{THEORETICAL PERFORMANCE OF COLLISION AVOIDANCE BY BRAKING}

We shall now specifically study what can be achieved by autonomous brake intervention. In order to avoid faulty interventions braking is only allowed when a collision becomes unavoidable i.e. the probability of collision is 1 . To get a feeling for what performance that is possible to achieve we look at one specific scenario. The scenario studied is a head on collision with a stationary object (see fig. 4 below).

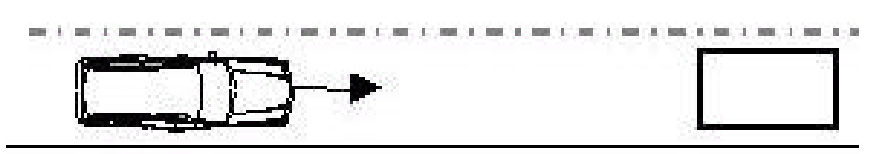

Figure 4. Car on collision course with a stationary obstacle

The braking distance and the distance needed to avoid the obstacle by steering away at different speeds is shown in figure 5 below

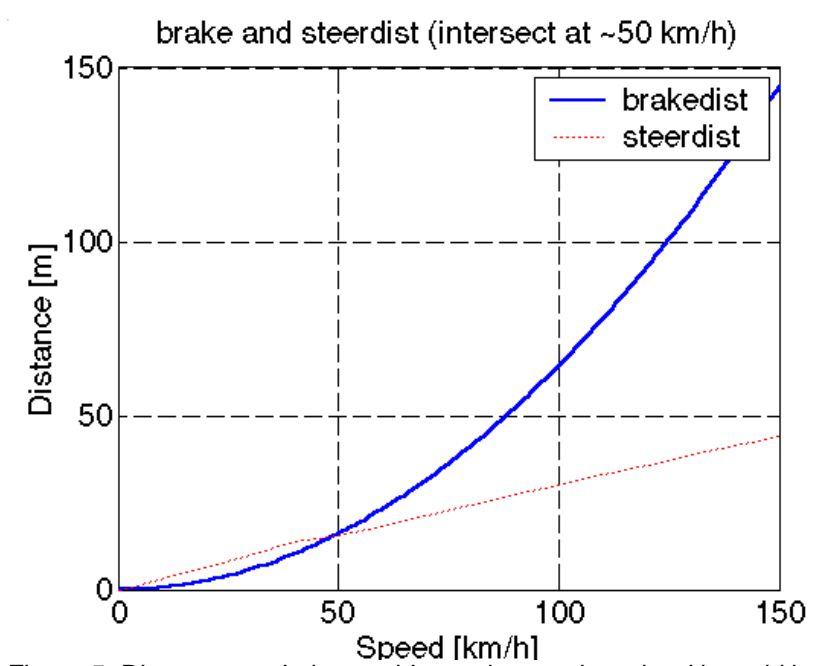

Figure 5. Distance needed to avoid a stationary obstacle with a width of 2 $\mathrm{m}$ by means of braking and steering.

We see in figure 5 that the braking distance (solid) and the steering away distance (dotted) cross somewhere around $50 \mathrm{~km} / \mathrm{h}$. This means that for low speeds, braking is the most efficient countermeasure and for high speeds steering away is more efficient. If full braking is applied at the point were collision becomes unavoidable (for low speeds at the braking distance and for high speeds at the steer away distance, then the collision speed as a function of the initial speed will be according to figure 6 below 


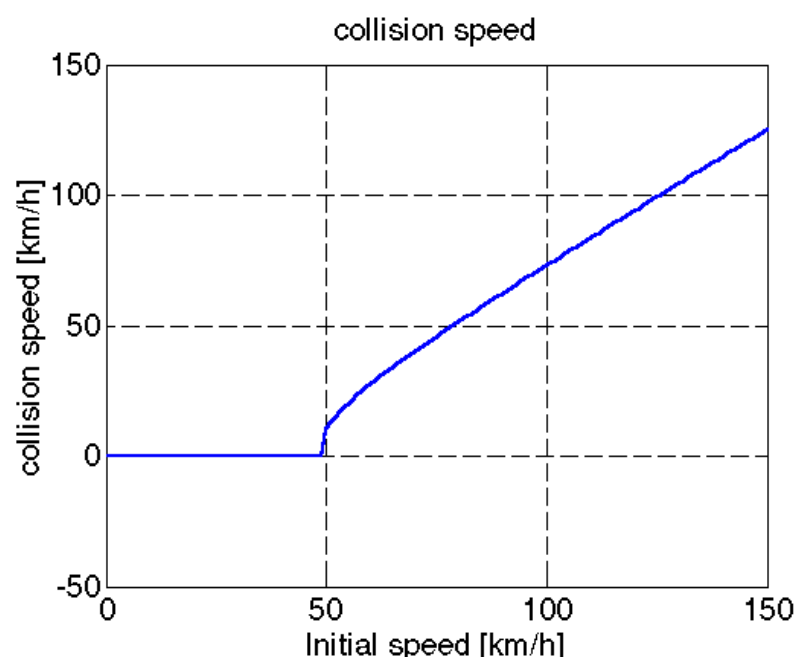

Figure 6. Collision speed when full braking is applied at the point where the collision becomes unavoidable.

\section{SIMULATON RESULTS}

To evaluate performance of the algorithm we have designed and simulated 30 different scenarios. The scenarios can be divided in to two groups. The first group of scenarios is designed to provoke the system to make a faulty intervention (= intervene when no collision occurs). The second group is designed to evaluate performance of the system in a situation where it should intervene i.e. when a collision occurs. Here we describe and present results from a selection of the simulated scenarios (all scenarios were simulated at speeds ranging from $10-150 \mathrm{~km} / \mathrm{h}$ ):

Symbols:

: The CMBB vehicle

$\square$ : The POV (Principal Other Vehicle)

\section{GROUP ONE SCENARIOS:}

These scenarios were designed to provoke a faulty intervention. The scenarios were simulated 10 times at each speed (speeds [10 20 30... 150]). From the simulation results no faulty interventions were observed.

\section{Scenario 1:}

Head to Head, the CMBB vehicle turns right at the last moment.

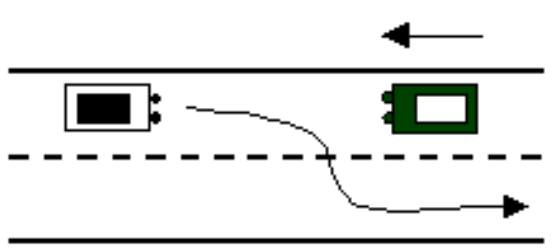

Figure 7. Scenario 1

\section{Scenario 2:}

Straight roadway, POV travelling in the same lane as the CMBB vehicle. Suddenly the POV brakes hard and then turns hard. The POV just clears the path of the $\mathrm{CMBB}$ vehicle.

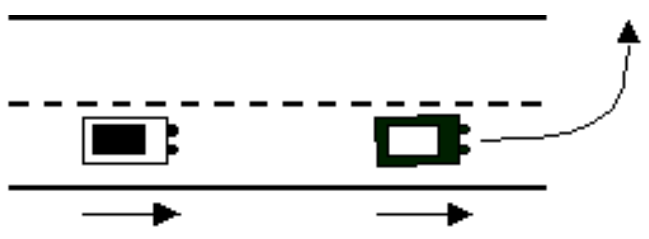

Figure 8. Scenario 2

Scenario 3:

The CMBB vehicle changes from right to left lane (both lanes in the same direction) at the same time as it meets another vehicle in the opposite lane.

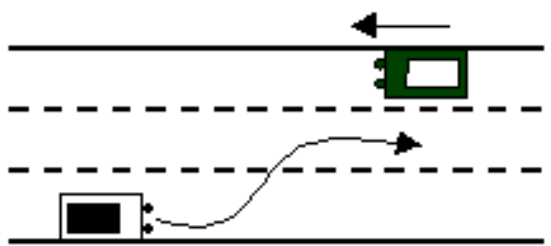

Figure 9. Scenario 3

\section{GROUP 2 SCENARIOS:}

The group 2 scenarios presented below are used to see what performance the system achieves.

\section{Scenario 4:}

Straight roadway, POV traveling in the same lane as the CMBB vehicle. Suddenly the POV brakes hard (deceleration $7 \mathrm{~m} / \mathrm{s}^{2}$ ). The headway is $15 \mathrm{~m}$. The initial speed, prior to the POV brake maneuver, was the same for both vehicles. 


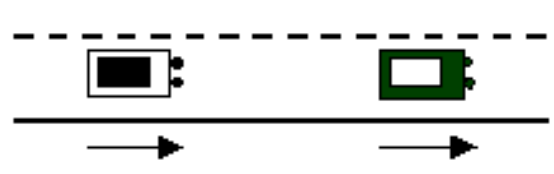

Figure 10. Scenario 4

Result: In figure 11 the relative speed at impact is plotted as a function of the initial speed. One can see that for low speeds the relative velocity at impact is reduced $\sim 10-20 \mathrm{~km} / \mathrm{h}$. For higher speeds the system response time is too long to be able to reduce the collision speed.

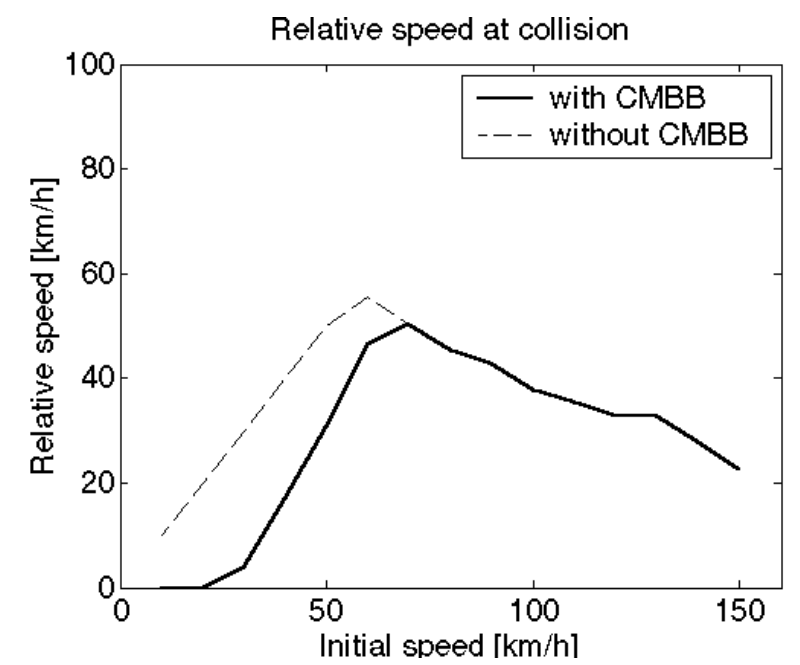

Figure 11. Relative speed at collision for scenario 4.

\section{Scenario 5:}

This scenario is the same as scenario 4 with the exception that there is a lateral offset of $0.5 \mathrm{~m}$ between the CMBB vehicle and the POV.

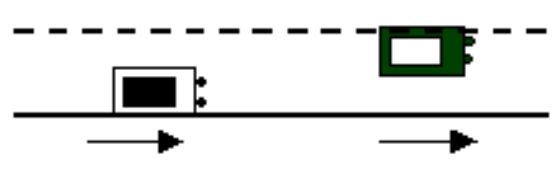

Figure 12. Scenario 5

Result: Again the relative speed of the vehicles at impact is plotted as a function of the initial speed (in figure 13). For comparison the results from the previous scenario has been plotted in figure 13. In the figure one can see that there is no intervention for low speeds. The reason for this is that before the decision is made the target moves out of the sensors' field of view.

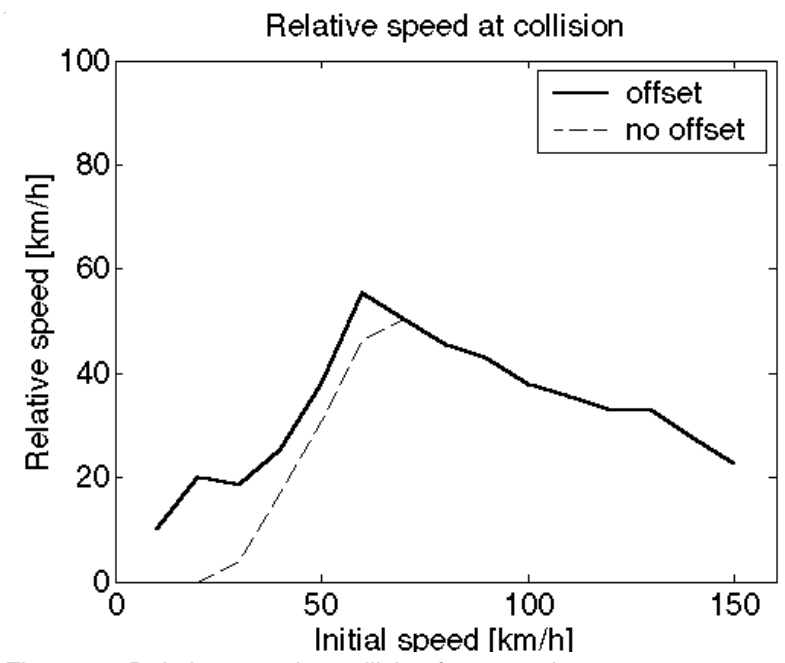

Figure 13. Relative speed at collision for scenario 5.

Scenario 6:

Object is on the side of the road and then suddenly "jumps" out $10 \mathrm{~m}$ in front of the CMBB vehicle.

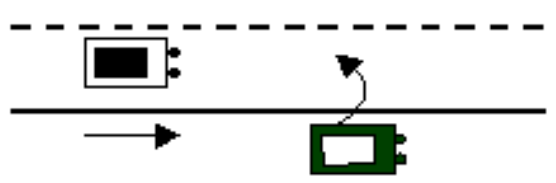

Figure 14. Scenario 6

The performance for scenario 6 is plotted as a phase diagram in figure 15. This shows impact speed and at what distance the system intervenes.

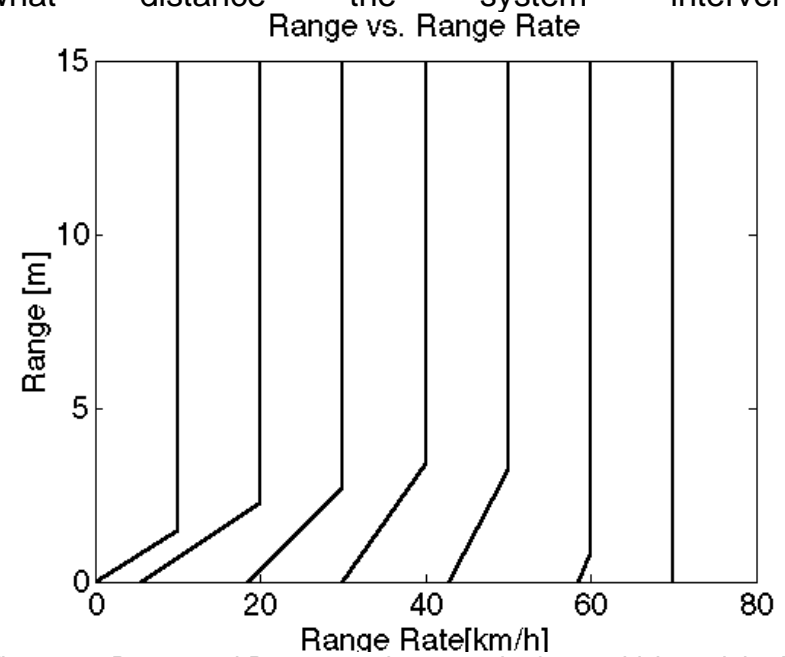

Figure 15. Range and Range rate between the host vehicle and the POV for scenario 6 . The results are based on simulated data. 


\section{TEST RESULTS}

The test vehicle is a Volvo V70 equipped with a millimeter wavelength radar and a laser radar. The sensor update rate is $10 \mathrm{~Hz}$ for both sensors and their field of view is $\sim 8$ degrees. The sensor fusion, data association and decision making algorithm execute on an on-board processing unit which is also connected to the vehicle's braking system. The purpose of having two sensors is to try to discriminate targets that are not valid. For example a millimeter wavelength radar can receive a strong echo from a tin can.

\section{CORRECTNESS OF THE ALGORITM}

To check if the algorithm makes faulty decision the prototype system has been driven in real traffic (urban and highway traffic) with braking disabled. We found that some faulty interventions do occur. These interventions mainly occur at low speeds when there are a lot of potential targets/obstacles close to the sensors (i.e. in front of the car). However we did not find any case where it was obvious that the algorithm made an erroneous decision based on a target that it had been tracking for some samples. All the faulty interventions seem to come from erroneous measurements, "false targets" or from bad initialization of obstacles.

\section{PERFORMANCE OF THE ALGORITHM}

To evaluate collision mitigation performance, collision tests against a stationary obstacle have been performed. As an obstacle, an inflatable car is used (fig 16).

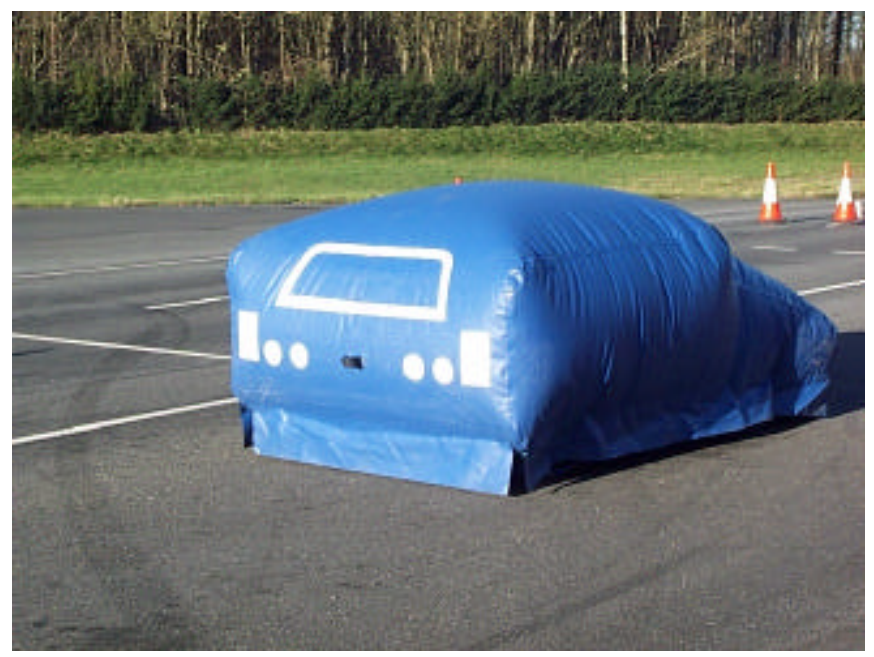

Figure 16. Inflatable car for testing system performance when colliding.

Performance for the head-on scenario pictured in figure 4 is plotted in figure 17. In figure 17 we plot range between host vehicle and obstacle vs. range rate, the plotted result is an average from 5-20 collisions at each speed. As can be seen in the figure, speed was reduced $10-17 \mathrm{~km} / \mathrm{h}$ for initial speeds ranging from $30-70 \mathrm{~km} / \mathrm{h}$.

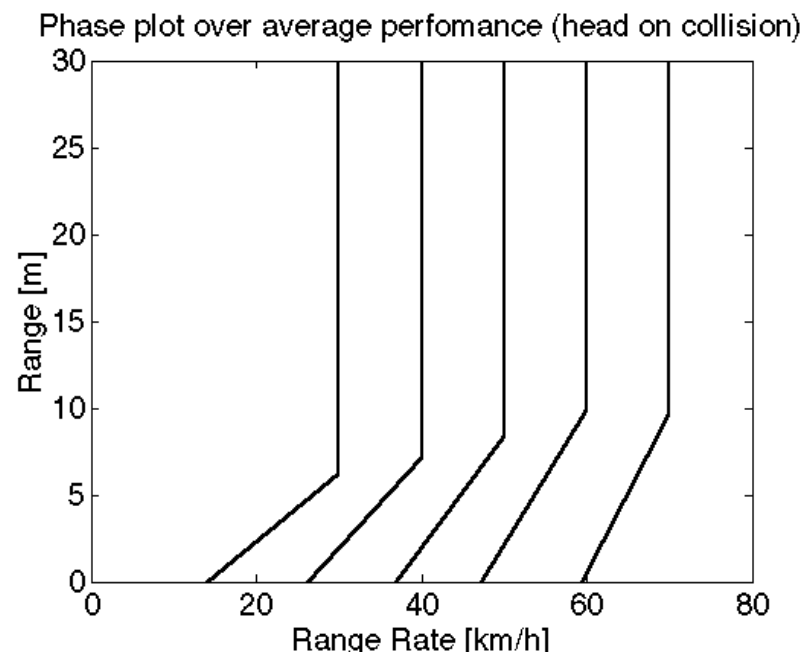

Figure 17. Range vs. Range Rate for the head on against stationary object scenario (figure 4). The result shown is the average performance from test drives with the V70 test vehicle.

\section{CONCLUSION}

A method for decision making in collision avoidance applications has been presented. The main advantages of the method are:

The use of modern tracking theory makes it straight forward how to deal with measurement and process noise

Motion in two dimensions is considered.

The prototype system presented in this paper significantly reduces the impact speed in frontal collisions. As can be seen in figure 17 interventions typically occur when the obstacle is closer than $20 \mathrm{~m}$ away from the CMBB vehicle (more than $90 \%$ of all rear end collisions occur at relative speeds below 100 $\mathrm{km} / \mathrm{h}$ [4]). A sensor with a shorter detection range but a larger field of view might be more appropriate for collision mitigation purposes.

Further work on the sensors and the sensorfusion is needed to have a system with 0 faulty interventions. It would be desirable to have a sensor with better target classification capability.

\section{DISCUSSION}

Differences between optimal performance (figure 5 and 6 ) and test result (figure 17) can be attributed to: 
Measurement uncertainties

System response time (computational time, sensor measurement rate)

System modeling errors

By improving these factors one can come closer to optimal performance. Some specific problems with the system presented here were that the laser radar and millimeter radar could not be synchronized. This of course causes some discrepancy between laser and radar measurement. Both sensor loses the target at close range $(<10 \mathrm{~m})$ because their narrow field of view. This cassias deteriorated performance at low speeds. The laser radar seem to have problem "seeing" the inflatable car which at some occasions caused missed interventions, since both sensors are required to detect the obstacle in order to have an intervention.

Another factor that limits the system performance is the time to build up brake pressure and maximum achieved pressure. The brake system used on the test vehicle here achieved decelerations between 5-7 $\mathrm{m} / \mathrm{s}^{2}$. A brake system that quickly gives a deceleration of $10 \mathrm{~m} / \mathrm{s}^{2}$ would give an additional speed reduction of $\sim 10 \mathrm{~km} / \mathrm{h}$ to the test results plotted in figure 17 .

To design a good collision avoidance system we need to solve two issues. One is the risk estimation discussed in this paper. The other issue, that has not been addressed here, is that of object recognition. This is a matter of the sensing capabilities of the sensors but also a matter of how to do the sensor fusion. For correct decision making accurate target classification and feature (for example width) extraction is imperative.

\section{REFERENCES}

1. Minoru Tamura, Hideaki Inoue, Takayuki Watnabe and Naoki Maruko. Research on a Brake Assist System with a Preview Function. SAE Paper 200101-0357

2. Development of an Adaptive Cruise Control with Stop-and-Go Capability, SAE Paper 2001-01-0807

3. Berthold Ulmer. VITA II - Active Collision Avoidance in Real Traffic. 1994

4. Bo Zhu. Potential Effects on Accidents from Forward Collision Warning/Avoidance System. Master thesis at Linköping University, LITH-ITN-EX150-SE, 2001.

5. Datta N. Godbole, Raja Sengupta, James Misener, Natasha Kourjanskaia, James B. Michael. (Transport Research record 1621,1998). Benefit Evaluation of Crash Avoidance System.

6. Farber E. I. Using the Reamacs Model to Compare the Effectiveness of Alternative Rear End Collision
Warning Algorithms. Conference on Enhanced Safety of Vehicle, Munich, Germany, 1994.

7. CAMP (Crash Avoidance Metric Partnership), Final report, 1999 ?.

8. H. C. Joksch. Velocity Change and Fatality Risk in a Crash-A Rule of Thumb. Accident Analysis and Prevention, vol25, No. 1, 1993.

9. J. R. Treat, N. S. McDonald, D. Shinar, R. D. Hume, R. E. Mayer, R. L. Stansifer and N. J. Castellan. Trilevel Study of the Cause of Traffic Accidents. Report No. DOT-HS-805-085, NHTSA, 1977.

10. R. Wade Allen (Transportation Research Record 1059) Research Methodology for Crash Avoidance Studies.

11. R. Wade Allen (VTI Report 331 A) Crash Avoidance Models and Driver/Vehicle Handling.

12. Ribert M. Clark, Michael J. Goodman. Michael Perel, and Ronald R. Knipling (DOT/FHWA, 1996) Driver Performance and IVHS Collision Avoidance Systems: A Search for Design-Relevant Measurement Protocol.

13. Ronald R. Knipling. (1993). IVHS Technologies Applied to Collision Avoidance: Perspectives on Six Target Crash Types and Countermeasures.

14. Thomas A. Dingus, Steven K. Jahns, Avraham D. Horowitz, and Ronal Knipling (Human Factors in Intelligent Transportation System, 1997). Human Factors Design Issues for Crash Avoidance System.

15. William A. Leasure. Jr. (1994) NHTSA's IVHS Collision Avoidance Research Program: Strategic Plan and Status Update.

16. F. Gustafson. Adaptive Filtering and Change Detection. John Wiley, 2000.

17. P. Barber, N. Clarke. Advanced Collision Warning Systems. Industrial Automation and Control: Applications in the Automotive Industry (Digest No. 1998/234).

\section{CONTACT}

\section{Jonas Jansson \\ Volvo Car Corporation \\ Dept 92421 \\ SE-405 31 Göteborg, Sweden \\ Telephone: +46 31595438 \\ E-mail: jiansso1@volvocars.com}

alt.

\section{Linköping University}

Division of Automatic Control

SE-581 83 Linköping, Sweden

Telephone: +46 13286697

E-mail: jansson@isy.liu.se 